\title{
Surface and curvature energies from jellium spheres: Density functional hierarchy and quantum Monte Carlo
}

\author{
L. M. Almeida* \\ Department of Physics, University of Aveiro, 3810 Aveiro, Portugal \\ John P. Perdew ${ }^{\dagger}$ \\ Department of Physics and Quantum Theory Group, Tulane University, New Orleans, Louisiana 70118
}

Carlos Fiolhais

Department of Physics and Center for Computational Physics, University of Coimbra, 3004-516 Coimbra, Portugal

(Received 7 February 2002; published 20 August 2002)

\begin{abstract}
We consider spherical jellium clusters with up to 200 electrons as a testing ground for density functional approximations to the exchange-correlation energy of a many-electron ground state. As nearly-exact standards, we employ Hartree-Fock energies at the exchange-only level and the diffusion Monte Carlo (DMC) energies of Sottile and Ballone (2001) at the correlated level. The density functionals tested are the local spin density (LSD), generalized gradient (GGA), and meta-generalized gradient (meta-GGA) approximations; the latter gives the most accurate results. By fitting the deviation from the LSD energy of closed-shell clusters to the predictions of the liquid drop model, we extract the exchange-correlation surface energies and curvature energies of a semi-infinite jellium from the energies of finite clusters. For the density functionals, the surface energies so extracted agree closely with those calculated directly for a single planar surface. But for the diffusion Monte Carlo method, the surface energies so extracted are considerably lower (and we suspect more accurate) than those extrapolated by Acioli and Ceperley (1996) from their DMC supercell calculations. The errors of the LSD, GGA, and meta-GGA surface and curvature energies are estimated, and are found to be consistently small for both properties only at the meta-GGA level. These errors are qualitatively related to relative performances of the various density functionals for the calculation of atomization energies: the proper self-interaction correction to the LSD for a one-electron atom is in the curvature energy (as it is in meta-GGA), not in the surface energy (as it is in GGA). Additionally, a formula is given for the interpolation and extrapolation of the surface energy $\sigma_{\mathrm{xc}}$ as a function of the bulk density parameter $r_{s}$.
\end{abstract}

DOI: 10.1103/PhysRevB.66.075115

PACS number(s): 71.15.Mb, 73.22.-f, 71.10.-w, 68.35.Md

\section{INTRODUCTION AND DENSITY FUNCTIONAL THEORY}

Kohn-Sham density functional theory ${ }^{1}$ is now perhaps the most widely used method of electronic structure calculation in both condensed matter physics and quantum chemistry. This self-consistent field theory would yield the exact ground state energy $E$ and spin densities $n_{\uparrow}(\mathbf{r})$ and $n_{\downarrow}(\mathbf{r})$ if the exact density functional $E_{\mathrm{xc}}\left[n_{\uparrow}, n_{\downarrow}\right]$ for the exchangecorrelation energy were known. The original local spin density (LSD) approximation is still widely used for solids, while more elaborate approximations have been developed for the more rapidly-varying electronic densities of atoms and molecules. While empirically constructed functionals can achieve the highest accuracy for limited classes of systems such as molecules, nonempirically constructed functionals tend to have a wider and more nearly universal range of application. ${ }^{2}$ Much remains to be done to test the functionals already developed, and to develop better ones.

Spherical jellium clusters ${ }^{3}$ are simple test systems which display both slowly- and rapidly-varying density regions as well as a wide range of density values. Although not very realistic, they are simple enough to be explored by wave function methods like diffusion Monte Carlo (DMC), which provide nearly-exact solutions to the many-electron Schrödinger equation. In the jellium model, $N$ interacting electrons are neutralized by a positive background of uniform density

$$
\bar{n}=3 / 4 \pi r_{s}^{3} .
$$

In the spherical jellium model of a metallic cluster, this background is contained inside a sphere of radius

$$
R=r_{s} N^{1 / 3}
$$

The ground-state energy $E$ and spin densities $n=n_{\uparrow}(\mathbf{r})$ and $n_{\downarrow}(\mathbf{r})$ can be found by solving the self-consistent KohnSham equations,

$$
\begin{aligned}
& \left(-\frac{1}{2} \nabla^{2}+v_{+}(\mathbf{r})+u([n] ; \mathbf{r})+v_{\mathrm{xc}}^{\sigma}\left(\left[n_{\uparrow}, n_{\downarrow}\right] ; \mathbf{r}\right)\right) \psi_{i \sigma}(\mathbf{r}) \\
& \quad=\epsilon_{i \sigma} \psi_{i \sigma}(\mathbf{r}),
\end{aligned}
$$

in atomic units where $\hbar=m=e^{2}=1$. The potential due to the positive background charge is

$$
v_{+}(r)= \begin{cases}-\frac{N}{2 R}\left[3-\left(\frac{r}{R}\right)^{2}\right] & (r \leqslant R) \\ -\frac{N}{r} & (r>R) .\end{cases}
$$

The Hartree potential is 


$$
u([n] ; \mathbf{r})=\int d^{3} r^{\prime} \frac{n\left(\mathbf{r}^{\prime}\right)}{\left|\mathbf{r}^{\prime}-\mathbf{r}\right|},
$$

where the total electron density is $n(\mathbf{r})=n_{\uparrow}(\mathbf{r})+n_{\downarrow}(\mathbf{r})$ $=\sum_{\sigma} n_{\sigma}(\mathbf{r})$, and

$$
n_{\sigma}(\mathbf{r})=\sum_{i}^{\text {occup }}\left|\psi_{i \sigma}(\mathbf{r})\right|^{2}
$$

The $\psi_{i \sigma}(\mathbf{r})$ are the occupied Kohn-Sham one-electron orbitals. The exchange-correlation potential is the functional derivative,

$$
v_{\mathrm{xc}}^{\sigma}\left(\left[n_{\uparrow}, n_{\downarrow}\right] ; \mathbf{r}\right)=\frac{\delta E_{x c}\left[n_{\uparrow}, n_{\downarrow}\right]}{\delta n_{\sigma}(\mathbf{r})} .
$$

Finally, the total energy is

$$
\begin{aligned}
E= & \sum_{i \sigma}^{\text {occup }}\left\langle\psi_{i \sigma}\left|-\frac{1}{2} \nabla^{2}\right| \psi_{i \sigma}\right\rangle+\int d^{3} r n(\mathbf{r}) v_{+}(\mathbf{r}) \\
& +\frac{1}{2} \int d^{3} \operatorname{rr}(\mathbf{r}) u([n] ; \mathbf{r})+\frac{3}{5} \frac{N^{2}}{R}+E_{\mathrm{xc}}\left[n_{\uparrow}, n_{\downarrow}\right],
\end{aligned}
$$

where the $3 N^{2} / 5 R$ term is the electrostatic self-energy of the positive background.

The local spin density (LSD) approximation ${ }^{1}$ is

$$
E_{\mathrm{xc}}^{\mathrm{LSD}}\left[n_{\uparrow}, n_{\downarrow}\right]=\int d^{3} r n(\mathbf{r}) \epsilon_{\mathrm{xc}}^{\mathrm{unif}}\left(n_{\downarrow}(\mathbf{r}), n_{\uparrow}(\mathbf{r})\right),
$$

where $\epsilon_{\mathrm{xc}}^{\mathrm{unif}}\left(n_{\downarrow}, n_{\uparrow}\right)$ is the exchange-correlation energy per electron of an electron gas with uniform spin densities $n_{\downarrow}$ and $n_{\uparrow}$. By construction, LSD is correct for slowly-varying $n_{\sigma}(\mathbf{r})$. We have adopted the parametrization for $\epsilon_{\mathrm{xc}}^{\mathrm{unif}}\left(n_{\downarrow}, n_{\uparrow}\right)$ of Perdew and Wang (1992), ${ }^{4}$ based upon the released-node diffusion Monte Carlo calculation of Ceperley and Alder ${ }^{5}$ for the uniform electron gas.

To better describe realistic density variations, semilocal or fully nonlocal density functionals have been developed. The most popular of these is the generalized gradient approximation (GGA), which makes use of the density gradient,

$$
\begin{gathered}
E_{\mathrm{xc}}^{\mathrm{GGA}}\left[n_{\uparrow}, n_{\downarrow}\right]=\int d^{3} r f_{\mathrm{xc}}\left(n_{\downarrow}, n_{\uparrow}, \nabla n_{\downarrow}, \nabla n_{\uparrow}\right), \\
v_{\mathrm{xc}}^{\sigma, \mathrm{GGA}}\left(\left[n_{\uparrow}, n_{\downarrow}\right] ; \mathbf{r}\right)=\frac{\partial f_{\mathrm{xc}}}{\partial n_{\sigma}}-\nabla \cdot\left(\frac{\partial f_{\mathrm{xc}}}{\partial\left(\nabla n_{\sigma}\right)}\right) .
\end{gathered}
$$

We consider two versions of GGA, corresponding to different choices for the function $f_{\mathrm{xc}}$; the semiempirically constructed Becke-Lee-Yang-Parr ${ }^{6,7}$ functional and the nonempirically-constructed Perdew-Burke-Ernzerhof (PBE) (Ref. 8) functionals. Of these, only the latter correctly reduces to LSD in the limit of a uniform density $\left(\nabla n_{\downarrow}\right.$ $\left.=\nabla n_{\uparrow}=0\right)$.

The PBE GGA satisfies more exact constraints on $E_{\mathrm{xc}}\left[n_{\downarrow}, n_{\uparrow}\right]$ than LSD does. Even more exact constraints can be satisfied by a meta-generalized gradient approximation (meta-GGA or MGGA)

$$
E_{\mathrm{xc}}^{\mathrm{MGGA}}\left[n_{\uparrow}, n_{\downarrow}\right]=\int d^{3} r f_{\mathrm{xc}}\left(n_{\uparrow}, n_{\downarrow}, \nabla n_{\uparrow}, \nabla n_{\downarrow}, \tau_{\uparrow}, \tau_{\downarrow}\right),
$$

which makes use not only of the GGA ingredients but also of the orbital kinetic energy densities,

$$
\tau_{\sigma}(\mathbf{r})=\frac{1}{2} \sum_{i}^{\text {occup }}\left|\nabla \psi_{i \sigma}(\mathbf{r})\right|^{2}
$$

We will consider the Perdew-Kurth-Zupan-Blaha (PKZB) (Ref. 9) meta-GGA, which is constructed in a largely nonempirical way but has one empirical parameter fitted to molecular atomization energies in its exchange component. Like the PBE GGA, the PKZB meta-GGA correctly reduces to LSD in the limit of a uniform density.

Part of our purpose is to test these functionals in comparison with the recent fixed-node diffusion Monte Carlo (DMC) calculations for closed-shell jellium spheres of Sottile and Ballone. ${ }^{10}$ These DMC calculations, which follow and complete previous work, ${ }^{11}$ are essentially exact apart from fixednode and statistical errors. Because they are made for finite systems, they are of course free from the size-extrapolation errors that may be present in DMC results for infinite ${ }^{5}$ or semi-infinite $^{12}$ systems. Our remaining purpose is to extract accurate jellium surface energies from these DMC results, since the "exact" values are controversial. ${ }^{12,13}$ In Sec. II, we will discuss the size effects that arise in jellium spheres, and will explain how we perform our calculations and how we extract surface energies. In Sec. III, we will present our results. Our conclusions will be summarized in Sec. IV.

Our focus is of course on the exchange-correlation contribution to the total surface energy $\sigma$. The exchangecorrelation surface energy $\sigma_{\mathrm{xc}}$ is not only the part of $\sigma$ that must be approximated, but its magnitude is typically somewhat greater than that of $\sigma$.

\section{SIZE EFFECTS AND EXTRACTION OF THE SURFACE ENERGY}

The energy of a large $(N \rightarrow \infty)$ neutral $N$-electron jellium sphere (and each of its kinetic, total electrostatic, exchange, and correlation components) is given by the liquid drop model (LDM), ${ }^{14}$

$$
\begin{aligned}
& E^{\mathrm{LDM}}=\frac{4 \pi}{3} R^{3} \alpha+4 \pi R^{2} \sigma+2 \pi R \gamma \\
& =\epsilon^{\mathrm{unif}} N+4 \pi r_{s}^{2} \sigma N^{2 / 3}+2 \pi r_{s} \gamma N^{1 / 3},
\end{aligned}
$$

where $\alpha, \sigma$, and $\gamma$ are parameters describing the volume, surface, and curvature energies respectively, and $\epsilon^{\text {unif }}$ $=\left(4 \pi r_{s}^{3} / 3\right) \alpha$ is the energy per electron of the uniform electron gas. The surface energy $\sigma$ of jellium was formulated within the LSD in Ref. 15 and the curvature energy $\gamma$ in Ref. 16. The energy per electron from Eq. (12) is then

$$
\frac{E^{\mathrm{LDM}}}{N}=\epsilon^{\mathrm{unif}}+4 \pi r_{s}^{2} \sigma N^{-1 / 3}+2 \pi r_{s} \gamma N^{-2 / 3} \text {. }
$$


The LDM neglects quantum energy oscillations due to shell structure which are important for the small clusters $(N \leqslant 200)$ we shall study. The true energy per electron is not a smooth function of $N$, like Eq. (13), but an oscillating function with local minima at the shell-closing magicnumbers $N=2,8,18,20,34,40,58,92,106, \ldots$. This oscillation arises from the structure of the Kohn-Sham orbitals, which are functionals of the density. For the sequence of closed-shell structures, the oscillation is presumably about the same in LSD as it is at any higher level of theory, and so cancels out of the difference. Thus our LDM equation for the closed-shell clusters, including the smaller ones, is

$$
\begin{aligned}
\frac{E}{N}-\frac{E^{\mathrm{LSD}}}{N}= & \left(\epsilon^{\mathrm{unif}}-\epsilon_{\mathrm{PW} 92}^{\mathrm{unif}}\right)+4 \pi r_{s}^{2}\left(\sigma-\sigma^{\mathrm{LSD}}\right) N^{-1 / 3} \\
& +2 \pi r_{s}\left(\gamma-\gamma^{\mathrm{LSD}}\right) N^{-2 / 3}
\end{aligned}
$$

where we have allowed for the possibility that the bulk energy per electron may differ from the PW92 paramerization used in our LSD calculations. [Our key assumption behind Eq. (14), confirmed below for jellium spheres, appears to be true as well for carbon fullerenes; see Fig. 3 of Ref. 17]. When we apply Eq. (14) in this article, we will always apply it to the exchange-correlation component of $E$, i.e., to $E_{\mathrm{xc}}$.

Since the LSD surface energy $\sigma^{\mathrm{LSD}}\left(r_{s}\right)$ is known from independent calculations for the single planar surface, ${ }^{15,16}$ we can extract the surface energy $\sigma$ at any higher level of theory (including GGA, meta-GGA, and DMC) by calculating the energies per electron beyond and within LSD and then fitting the difference as a function of $N$ to Eq. (14), treating $\epsilon^{\text {unif }}$ $-\epsilon_{P W 92}^{\mathrm{unif}}, \sigma-\sigma^{\mathrm{LSD}}$, and $\gamma-\gamma^{\mathrm{LSD}}$ as the fit parameters. But since the quantity $\epsilon^{\text {unif }}-\epsilon_{\mathrm{PW} 92}^{\text {unif }}$ is also known at least approximately (and vanishes within PBE GGA and PKZB metaGGA), we actually constrain this parameter and find only $\sigma-\sigma^{\mathrm{LSD}}$ and $\gamma-\gamma^{\mathrm{LSD}}$ by fitting.

The logical consistency of Eq. (14) depends in part upon the assumption that the electron density changes little from LSD to any higher level of theory. Figure 1 shows that the GGA (PBE) density of a spherical jellium cluster is in fact very close to the LSD density. This is not a surprising conclusion: it also holds for the LSD and GGA densities of atoms and molecules, ${ }^{18}$ and for the LSD and DMC densities of spherical jellium clusters. ${ }^{10}$

Table I shows the very small energy differences between fully self-consistent GGA and "post-LSD" GGA, in which the GGA energy is evaluated for the LSD density. The energy effects of full self-consistency beyond post-LSD are typically negligible. ${ }^{18,19}$ Thus, in the rest of this work, we calculate GGA and meta-GGA energies on LSD densities, and we extract the DMC exchange-correlation energy by subtracting the LSD kinetic and electrostatic energies from the DMC total energy. Since the DMC total energy is variationally insensitive to the small difference between the DMC and LSD density profiles, we are effectively comparing the exchange-correlation energies predicted for the same LSD density by various density functionals and by DMC; this is a valid comparison. If we used instead the self-consistent GGA density profiles, all our exchange-correlation energies would

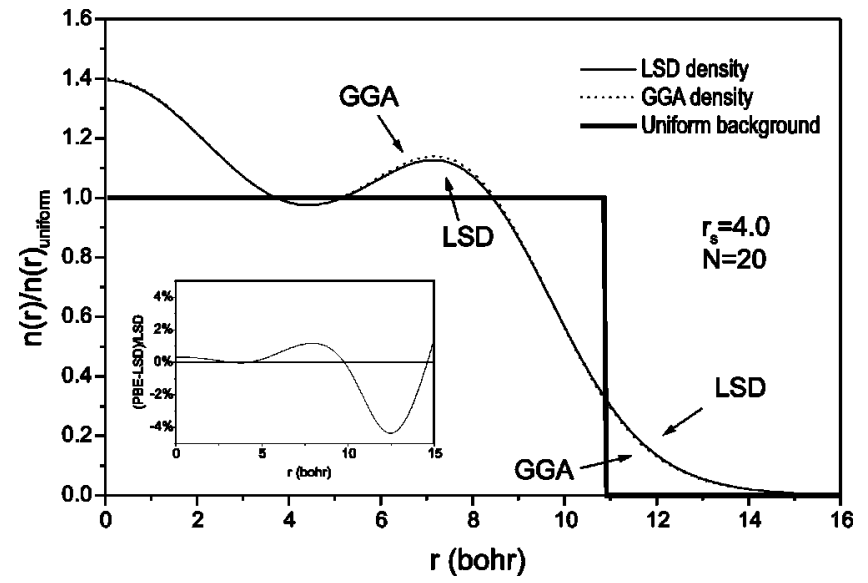

FIG. 1. Electron density from self-consistent density functional calculations in the local spin density approximation or LSD and in the generalized gradient approximation GGA(PBE) for a jellium sodium $\left(r_{s}=4.00\right)$ sphere with 20 electrons. Inset: relative differences of GGA and LSD densities. Note that GGA favors density inhomogeneity slightly more than LSD does.

change slightly, but in about the same way, leaving our conclusions unchanged. For the single planar surface of $r_{s}$ $=4.00$ jellium, using the LSD and GGA density profiles, the surface exchange-correlation energies are in PBE GGA 252.5 and $243.5 \mathrm{erg} / \mathrm{cm}^{2}$, and in PKZB meta-GGA 265.6 and $257.5 \mathrm{erg} / \mathrm{cm}^{2}$, respectively. Similar small shifts are found in the surface energies we extract from jellium spheres.

We are interested in the closed shell clusters, in which the electron density is naturally spherically symmetric and spinunpolarized. When we occasionally consider open-shell clusters, we construct a spherically symmetric spin-independent Kohn-Sham potential in the following way: We replace the spherical harmonic factor $Y_{l m}(\theta, \phi)$ by $Y_{00}=1 / \sqrt{4 \pi}$ in each Kohn-Sham orbital of Eq. (4), and we replace $n_{\uparrow}(r)$ and $n_{\downarrow}(r)$ by $n(r) / 2$ in the exchange-correlation potential of Eq. (5). Occupying the Kohn-Sham orbitals according to Hund's rule, we find a spherically-symmetric but possibly spinpolarized LSD density on which we calculate the LSD, GGA, or meta-GGA energy. In addition to those calculations

TABLE I. Comparison of energies for jellium sodium $\left(r_{s}\right.$ $=4.00$ ) spheres with $N=18$ and 20 electrons. The LSD calculation is fully self-consistent. The GGA(PBE) calculation is done fully self-consistently (SC-GGA) and also post-LSD (PLSD-GGA), i.e., using the LSD density.

\begin{tabular}{lcccc}
\hline \hline \multicolumn{5}{c}{ Energy $($ hartree) } \\
$N$ & & LSD & SC-GGA & PLSD-GGA \\
\hline 18 & $E$ & -1.2361 & -1.2481 & -1.2478 \\
18 & $E_{x}$ & -1.8724 & -1.9726 & -1.9669 \\
18 & $E_{c}$ & -0.5388 & -0.4569 & -0.4560 \\
& & & & \\
20 & $E$ & -1.3858 & -1.3980 & -1.3977 \\
20 & $E_{x}$ & -2.0663 & -2.1688 & -2.1628 \\
20 & $E_{c}$ & -0.5967 & -0.5131 & -0.5121 \\
\hline \hline
\end{tabular}


TABLE II. Total energies of jellium spheres with 20 electrons for different background densities. The results of the density functional approaches are compared to the fixed-node diffusion Monte Carlo or DMC values. Mean absolute differences ("mad") from DMC are presented in the last row. In this and subsequent tables and figures, all the density functionals are evaluated post-LSD (as defined in the caption of Table I). We show results for LSD, two GGA's (BLYP and PBE) and one meta-generalized gradient approximation (PKZB).

\begin{tabular}{lccccc}
\hline \hline \multicolumn{5}{c}{ Energy } & (hartree) for $N=20$ \\
$r_{s}$ (bohr) & LSD & BLYP & PBE & PKZB & DMC $^{\mathrm{a}}$ \\
\hline 0.30 & 99.175 & 99.056 & 98.967 & 99.134 & 99.107 \\
0.50 & 38.279 & 38.284 & 38.136 & 38.233 & 38.170 \\
1.00 & 7.583 & 7.7057 & 7.5078 & 7.5465 & 7.5197 \\
2.00 & -0.177 & 0.0117 & -0.2096 & -0.1987 & -0.1927 \\
3.25 & -1.2956 & -1.0917 & -1.3121 & -1.3095 & -1.2938 \\
4.00 & -1.3858 & -1.1826 & -1.3977 & -1.3970 & -1.3800 \\
5.62 & -1.3183 & -1.1244 & -1.3249 & -1.3259 & -1.3095 \\
mad & 0.039 & 0.163 & 0.036 & 0.025 & \\
\hline \hline
\end{tabular}

${ }^{\mathrm{a}}$ Reference 10.

for spherical jellium clusters, we have also performed postLSD density functional calculations for the single planar jellium surface. ${ }^{15}$

\section{RESULTS AND ANALYSIS}

The energies of neutral jellium spheres with $N \leqslant 200$ were calculated within LSD, GGA, and meta-GGA, as explained in Sec. I and II.

Table II shows the total energies $E$ for spheres with $N$ $=20$ electrons, for several values of the background density parameter $r_{s}$. In comparison with DMC, the least accurate results are found with the BLYP GGA (which predicts an incorrect correlation energy for the uniform gas), and the most accurate results (on average) are found with the PKZB meta-GGA. Table III shows $E / N$ vs $N$, the raw data of our analysis, at $r_{s}=4.00$.

Table IV displays the errors of the various density functionals for the total energy per electron $E / N$, averaged over the closed-shell clusters with $2 \leqslant N \leqslant 106$. Again the BLYP errors are much larger even than those of LSD. The PBE GGA errors are slightly smaller than those of LSD, and the PKZB meta-GGA errors are significantly smaller.

Table $\mathrm{V}$ displays the relative errors in the correlation energies, averaged over the closed-shell clusters. Here we see a clear improvement when we pass from LSD to GGA (PBE) or meta-GGA (PKZB). The improvement from LSD to GGA (PBE) was not so evident in Table II because of the strong cancellation of error between exchange and correlation which occurs in all the density functionals, but especially in LSD.

By subtracting the correlation from the total energy, we obtain an exchange-only total energy that can be compared to the Hartree-Fock (HF) energy. Figure 2 shows that this exchange-only total energy is much closer to the HF total energy $^{20}$ in the PKZB meta-GGA than it is in LSD. The
TABLE III. Total energies per electron of jellium spheres at $r_{s}$ $=4.00$ for different magic clusters. The results of the density functional approaches are compared to the fixed-node diffusion Monte Carlo or DMC values. Mean absolute differences ("mad") from DMC are presented in the last row. Note that the DMC energies are probably a little higher than exact energies, because of the fixednode error.

\begin{tabular}{lccccc}
\hline \hline \multicolumn{5}{c}{ Energy/N } & (hartree) for $r_{s}=4.00$ \\
$N$ & LSD & BLYP & PBE & PKZB & DMC $^{\mathrm{a}}$ \\
\hline 2 & -0.0617 & -0.0591 & -0.0635 & -0.0638 & -0.0641 \\
8 & -0.0672 & -0.0594 & -0.0681 & -0.0684 & -0.0674 \\
18 & -0.0687 & -0.0589 & -0.0693 & -0.0692 & -0.0684 \\
20 & -0.0693 & -0.0591 & -0.0699 & -0.0698 & -0.0690 \\
34 & -0.0704 & -0.0594 & -0.0709 & -0.0707 & -0.0700 \\
40 & -0.0702 & -0.0588 & -0.0707 & -0.0705 & -0.0697 \\
58 & -0.0718 & -0.0600 & -0.0722 & -0.0720 & -0.0713 \\
92 & -0.0727 & -0.0603 & -0.0730 & -0.0729 & -0.0721 \\
106 & -0.0717 & -0.0591 & -0.0720 & -0.0718 & -0.0710 \\
mad & 0.0007 & 0.0099 & 0.0009 & 0.0007 & \\
\hline \hline
\end{tabular}

${ }^{\mathrm{a}}$ Reference 10 .

dependence of the exchange energy upon the density is strong nonlocal, and much of this nonlocality is captured by the meta-GGA.

Reference 2 showed that the PKZB meta-GGA accurately describes the surface exchange energy of a semi-infinite jellium. Table VI shows that the exchange-only total surface energy is also accurately predicted by the meta-GGA, in comparison with Hartree-Fock values. ${ }^{21}$

Figure 3 shows the deviations from the LSD of the PBE GGA and PKZB meta-GGA exchange-correlation energies $E_{\mathrm{xc}}$. The GGA and meta-GGA deviations are similar for $N$ $\leqslant 30$, but the meta-GGA deviations are considerably smaller than the GGA ones at larger $N$. While the PBE GGA corrections to LSD have a slight tendency to stabilize open shells relative to closed ones, the PKZB meta-GGA corrections have a much stronger tendency to do so for the larger clusters.

Figure 4 shows how the shell-structure oscillations in $E_{\mathrm{xc}} / N-\epsilon_{\mathrm{xc}}^{\text {unif }}$ damp out as $N \rightarrow \infty\left(N^{-1 / 3} \rightarrow 0\right)$ for jellium spheres, and how this energy difference approaches

TABLE IV. Mean absolute deviations from fixed-node DMC values (Ref. 10) of the total energies per electron in various density functional approaches. The values are averages over nine magic clusters with $N$ electrons: $N=2,8,18,20,34,40,58,92$, and 106 .

\begin{tabular}{lcccc}
\hline \hline & \multicolumn{4}{c}{$\left|\left(E-E^{\mathrm{DMC}}\right) / N\right|$} \\
$r_{s}$ & LSD & BLYP & PBE & PKZB \\
\hline 1.00 & 0.0034 & 0.0093 & 0.0023 & 0.0010 \\
2.00 & 0.0015 & 0.0103 & 0.0013 & 0.0006 \\
3.25 & 0.0008 & 0.0101 & 0.0010 & 0.0007 \\
4.00 & 0.0007 & 0.0099 & 0.0009 & 0.0007 \\
5.62 & 0.0006 & 0.0092 & 0.0007 & 0.0007 \\
average & 0.0014 & 0.0095 & 0.0012 & 0.0007 \\
\hline \hline
\end{tabular}


TABLE V. Average relative deviations of correlation energies, within various density functional approaches, from DMC values (Ref. 10). Averages were taken over magic clusters: $N=2,8,18$, 20, 34, 40, 58, 92, and 106 .

\begin{tabular}{lcccc}
\hline \hline & \multicolumn{4}{c}{$\left(E_{c}-E_{c}^{\mathrm{DMC}}\right) / E_{c}^{\mathrm{DMC}}$} \\
$r_{s}$ & LSD & BLYP & PBE & PKZB \\
\hline 1.00 & $43.2 \%$ & $-20.7 \%$ & $9.0 \%$ & $9.7 \%$ \\
2.00 & $36.8 \%$ & $-28.1 \%$ & $10.1 \%$ & $10.2 \%$ \\
3.25 & $31.7 \%$ & $-35.4 \%$ & $9.1 \%$ & $8.7 \%$ \\
4.00 & $29.4 \%$ & $-38.8 \%$ & $8.2 \%$ & $7.7 \%$ \\
5.62 & $26.8 \%$ & $-43.6 \%$ & $7.6 \%$ & $6.7 \%$ \\
average & $33.6 \%$ & $-33.3 \%$ & $8.8 \%$ & $8.6 \%$ \\
\hline \hline
\end{tabular}

$4 \pi r_{s}^{2} \sigma_{\mathrm{xc}} N^{-1 / 3}$ as predicted by Eq. (13).

The key figure in this work is Fig. 5, which shows how the PBE, PKZB, and DMC jellium surface exchangecorrelation energy $\sigma_{\mathrm{xc}}$ and curvature energy $\gamma_{\mathrm{xc}}$ are extracted by fitting the left-hand side of Eq. (14) to the parabola of the right-hand side for the sequence of closed-shell clusters (excluding $N=2$ and 8). In this fit, $\epsilon_{\mathrm{xc}}^{\mathrm{unif}}-\epsilon_{x c, P W 92}^{\mathrm{unif}}$ is properly constrained to zero for PBE and PKZB. But the fixed-node DMC energies ${ }^{10}$ to which we fit must tend in the limit $N$ $\rightarrow \infty$ to values higher than the released-node DMC energies ${ }^{5}$ of the uniform gas which PW92 represents. For DMC, we take $\epsilon_{\mathrm{xc}}^{\text {unif }}=\epsilon_{x}^{\text {unif }}+\epsilon_{c, O B}^{\text {unif }}$, the Ortiz-Ballone ${ }^{22}$ parametrization of the fixed-node energy of the uniform gas ${ }^{22}$ (Table VII). We also tried another parametrization ${ }^{23}$ of the same energy, which did not affect our conclusions. In Fig. 5, we observe that the PKZB meta-GGA is much more like DMC than the PBE GGA is, both in initial slope at $N^{-1 / 3}=0$ and in curvature. From the fits, we find $\sigma_{\mathrm{xc}}-\sigma_{\mathrm{xc}}^{\mathrm{LSD}}$ (which is used to construct Table VIII) and also $\gamma_{\mathrm{xc}}-\gamma_{\mathrm{xc}}^{\mathrm{LSD}}$ (Table IX). Note that the curvature energy $\gamma_{\mathrm{xc}}$ is much lower in PKZB and DMC than it is in LSD or PBE.

Table VIII is the key table of this work. The first column

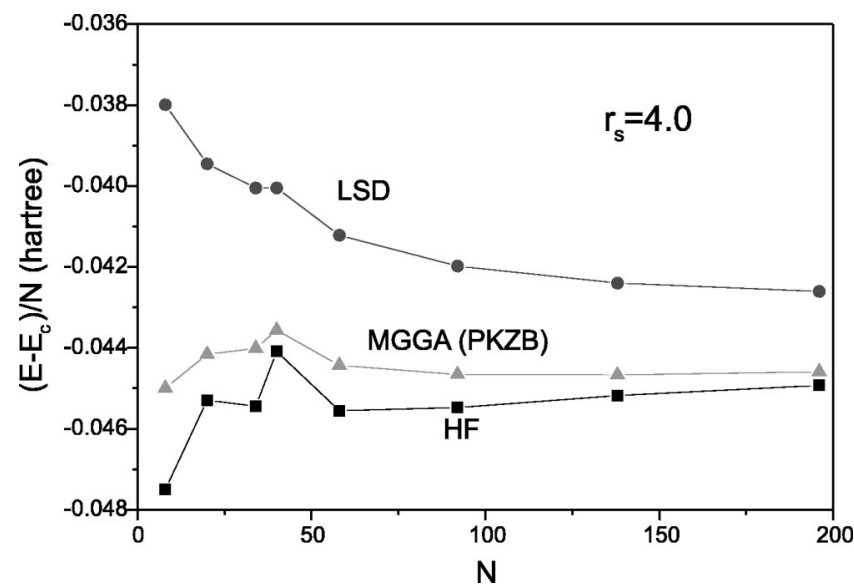

FIG. 2. Hartree-Fock (HF) total energies per electron (Ref. 20) compared to LSD and meta-generalized gradient approximation or MGGA exchange-only total energies $E-E_{c}$ for jellium spheres $\left(r_{s}=4.00\right)$. The values shown are for magic clusters with $N=8,20$, 34, 40, 58, 92, 138, and 196.
TABLE VI. Exchange-only total surface energies $\sigma-\sigma_{c}$ in LSD, GGA (PBE), and MGGA (PKZB), compared with the Hartree-Fock (HF) surface energy $\left(1\right.$ hartree/bohr ${ }^{2}=1.557$ $\left.\times 10^{6} \mathrm{erg} / \mathrm{cm}^{2}\right)$.

\begin{tabular}{lcccc}
\hline \hline \multicolumn{4}{c}{$\sigma-\sigma_{c}\left(\mathrm{erg} / \mathrm{cm}^{2}\right)$} \\
$r_{s}$ & LSD & PBE & PKZB & HF $^{\mathrm{a}}$ \\
\hline 2.07 & -894 & -1442 & -1316 & -1273 \\
2.30 & -314 & -729 & -640 & -674 \\
2.66 & 34 & -248 & -192 & -215 \\
3.28 & 147 & -14 & 13 & 5 \\
3.99 & 125 & 30 & 44 & 40 \\
4.96 & 80 & 27 & 34 & 35 \\
\hline \hline
\end{tabular}

${ }^{\mathrm{a}}$ Reference 21 .

is the LSD surface exchange-correlation energy of jellium, which we have calculated for a single planar surface. By adding $\sigma_{\mathrm{xc}}^{P B E}-\sigma_{\mathrm{xc}}^{\mathrm{LSD}}$ from the fit of Fig. 5 to $\sigma_{\mathrm{xc}}^{\mathrm{LSD}}$, we obtain the third column, $\sigma_{\mathrm{xc}}^{\mathrm{PBE}}$ fit , which agrees closely with $\sigma_{\mathrm{xc}}^{P B E}$ calculated for a single planar surface (fourth column). This procedure is repeated for PKZB, which again shows close agreement between $\sigma_{\text {xc }}^{\text {PKZB fit }}$ and $\sigma_{\text {xc }}^{P K Z B}$, and then for DMC.

While $\sigma_{\mathrm{xc}}^{\mathrm{DMC} \text { fit }}$ is rather close to $\sigma_{\mathrm{xc}}^{\mathrm{PKZB}}$, both are much lower than $\sigma_{\mathrm{xc}}^{D M C}$ from the planar surface DMC calculation of Acioli and Ceperley. ${ }^{12}$ We interpret this as further evidence that the surface energies of Ref. 12 are significantly too high, and that the PKZB surface energies are essentially correct, as suggested in recent work. ${ }^{13,24,34}$

The close agreement between $\sigma_{\text {xc }}^{\mathrm{PKZB}}$ fit and $\sigma_{\text {xc }}^{\mathrm{PKZB}}$ in Table VIII (and the similar agreement for PBE) gives us some confidence in our $\sigma_{\text {xc }}^{\text {DMC fit }}$ values. However, the accuracy of DMC fit is probably somewhat less than the precision of PKZB fit, for several reasons: (1) We have to assume [Eq. (14)] that corrections to the liquid drop model cancel out of the difference between the beyond-LSD and LSD energies. While this assumption has been confirmed to remarkable ac-

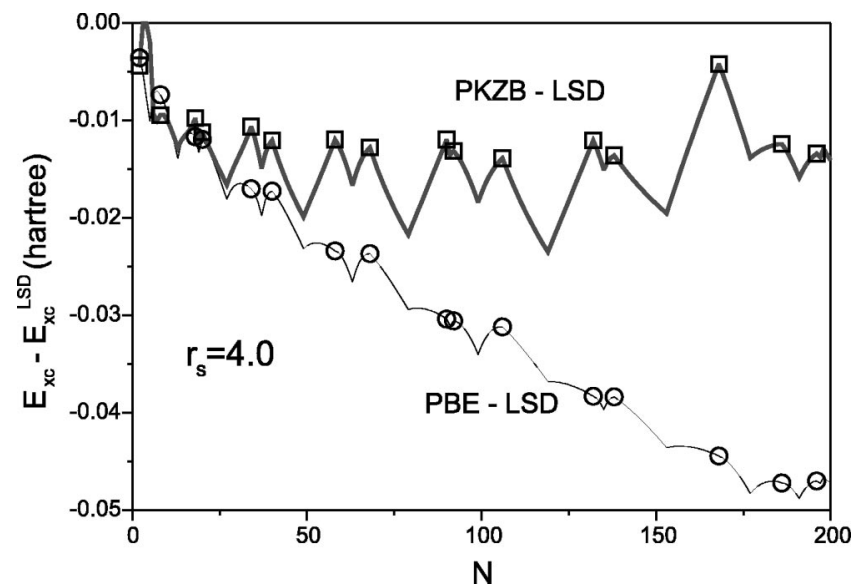

FIG. 3. Deviation of the exchange-correlation energy $E_{\mathrm{xc}}$ in PBE or PKZB from its LSD value for $r_{s}=4.00$ jellium spheres up to $N=200$, using LSD densities. The open circles in PBE-LSD and open squares in PKZB-LSD show the values for clusters with closed shells (magic clusters). 

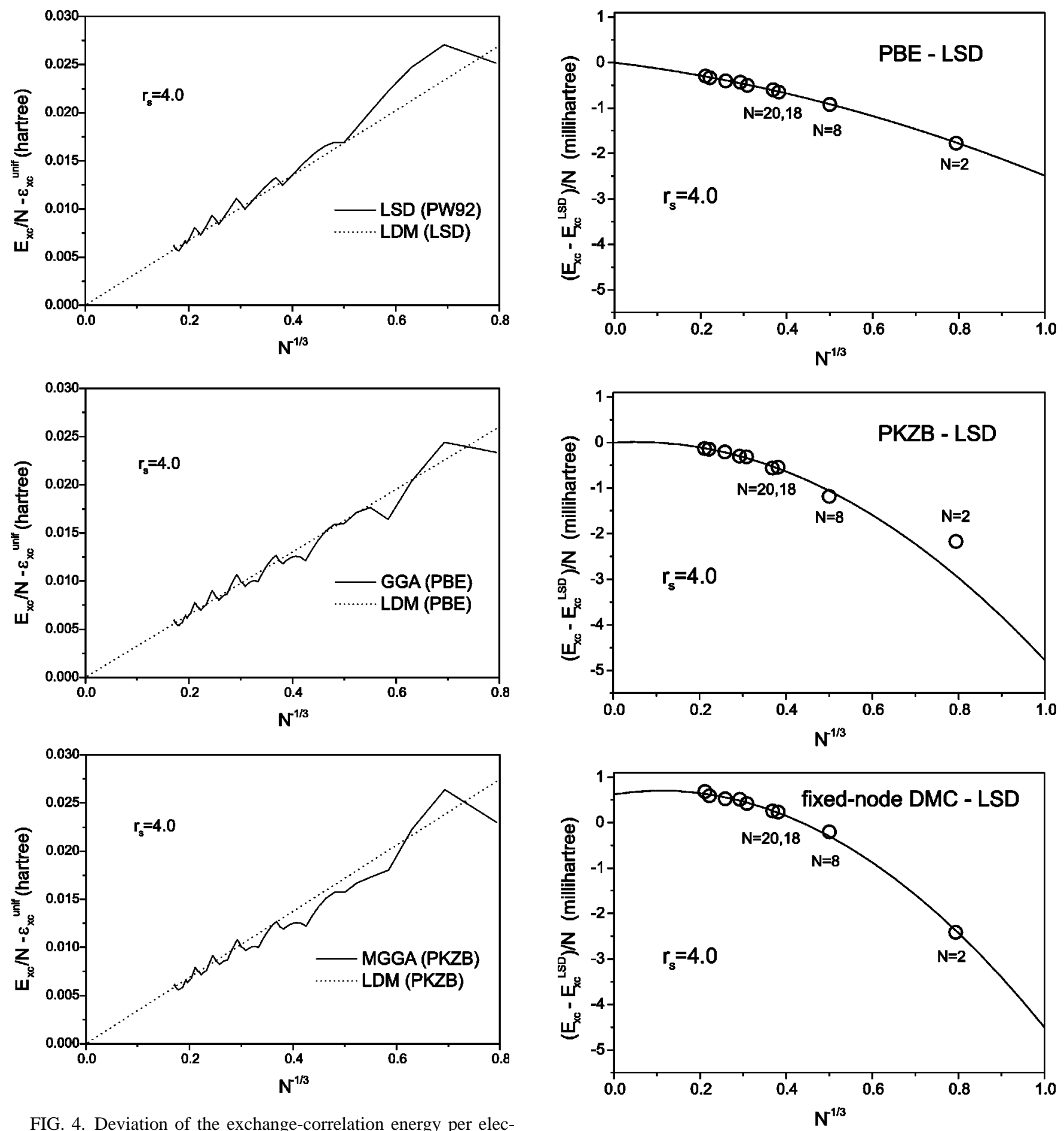

FIG. 4. Deviation of the exchange-correlation energy per electron, $E_{\mathrm{xc}} / N$, from its uniform-gas value $\epsilon_{\mathrm{xc}}^{\text {unif }}$, for LSD densities. Minima of this quantity tend to occur at closed-shell magic numbers. Comparison of LSD, GGA (PBE), and MGGA (PKZB) values for jellium spheres $\left(r_{s}=4.00\right)$ with the respective liquid drop model (LDM) values, $4 \pi r_{s}^{2} \sigma_{\mathrm{xc}} N^{-1 / 3}$ (including only the surface energy $\sigma_{\mathrm{xc}}$ and not the curvature energy).

curacy for PBE and PKZB, it is not necessarily true to the same extent for the difference between DMC and LSD. (2) The DMC energies for the jellium spheres contain fixed-node and statistical errors. Very small relative errors in the total energy become much larger errors in the surface energy. (3)

FIG. 5. Energy deviation from LSD of GGA (PBE), MGGA (PKZB), and DMC for jellium spheres with $r_{s}=4.00$ bohr. The full lines are parabolas fitted to the liquid drop model via Eq. (14), as explained in the text. The open circles are input values for $N=2,8$, $18,20,34,40,58,92$, and 106 . The first derivative of each parabola at $N^{-1 / 3}=0$ gives the corresponding fit correction to the LSD surface energy, while the second derivative at any $N^{-1 / 3}$ gives the correction to the LSD curvature energy. As explained near the end of Sec. II, all the exchange-correlation energies, including that of DMC, are effectively evaluated on the same LSD density. 
TABLE VII. Correlation energies per electron of the uniform electron gas in the Perdew-Wang (PW92) and Ortiz-Ballone (OB) parametrizations. Here $\Delta \epsilon_{c}^{O B}$ is the deviation from the PW92 parametrization, which may represent the fixed-node error.

\begin{tabular}{lccl}
\hline \hline & \multicolumn{3}{c}{$\epsilon_{c}^{\text {unif }}($ millihartree $)$} \\
$r_{s}$ & PW92 & OB $^{\text {a }}$ & $\Delta \epsilon_{c}^{O B}$ \\
\hline 1.00 & -59.774 & -58.028 & 1.746 \\
2.00 & -44.760 & -43.346 & 1.414 \\
3.25 & -35.489 & -34.601 & 0.887 \\
4.00 & -31.866 & -31.258 & 0.608 \\
5.62 & -26.408 & -26.276 & 0.132 \\
\hline \hline
\end{tabular}

${ }^{\mathrm{a}}$ Reference 22 .

The bulk limit $\epsilon^{\text {unif }}$ used in Eq. (14) should be the true limit of $E / N$ as $N \rightarrow \infty$. While we know the exact bulk limit $\epsilon_{\mathrm{xc}}^{\mathrm{unif}}$ for the PBE and PKZB $E_{\mathrm{xc}} / N$, we only have an estimate 22 for DMC, albeit an estimate acceptable to the second author of Refs. 10 and 22. We suspect that this third problem is the most severe one for our DMC fit. It should be even more severe for the DMC surface energies of Ref. 12, where apparently the bulk limit was taken to be a released-node DMC energy, inconsistent with the fixed-node DMC energies evaluated there for finite cells; this choice would overestimate the surface energy. For further discussion of the role of the bulk limit in surface energy calculations, see Refs. 25 and 26.

We believe that the most accurate jellium surface energies available are the RPA+ (Refs. 13,24,27) values, which provided the "exact" standard in Ref. 2. In RPA+, the exchange energy and the random phase approximation part of the correlation energy are treated exactly, and only the correction to RPA is treated in a nonempirical generalized gradient approximation. (An alternative correction to RPA is also under study. ${ }^{28,29}$ )

In the $r_{s} \rightarrow 0$ limit, the exchange-correlation energy becomes exchange-dominated and the density becomes slowlyvarying on the scale of $r_{s}$. In this limit, each of our density

TABLE VIII. Jellium surface exchange-correlation energies $\sigma_{\text {xc }}$ evaluated for LSD densities. Values calculated directly for a single planar surface are compared to those extracted from finite jellium spheres via "fits" like those of Fig. 5. The DMC values $\sigma_{\mathrm{HF}}$ $+\sigma_{c(Q M C)}-\left(\sigma_{\mathrm{LSD}}-\sigma_{\mathrm{xc} \mathrm{LSD}}\right)$ that we estimated from $\sigma_{\mathrm{HF}}$ and $\sigma_{c(\mathrm{QMC})}$ from Table $\mathrm{V}$ of Ref. 12 were $3153,1342,711$, and $394 \mathrm{erg} / \mathrm{cm}^{2}$ for $r_{s}=2.07,2.66,3.25$, and 3.93, respectively; these were interpolated to $r_{s}=2.00$ and 4.00 using our Eq. (15).

\begin{tabular}{lccccccc}
\hline \hline & & \multicolumn{6}{c}{$\sigma_{\mathrm{xc}}\left(\mathrm{erg} / \mathrm{cm}^{2}\right)$} \\
$r_{s}$ & LSD & PBE fit & PBE & PKZB fit & PKZB & DMC fit & DMC $^{\text {a }}$ \\
\hline 1.00 & 40928 & 40068 & 40276 & 41637 & 41463 & 41196 & \\
2.00 & 3357 & 3263 & 3263 & 3420 & 3400 & 3347 & 3566 \\
3.25 & 568.6 & 550.0 & 549.5 & 578.5 & 576.4 & 574.1 & 711 \\
4.00 & 261.7 & 252.6 & 252.5 & 265.9 & 265.6 & 272.8 & 372 \\
5.62 & 70.0 & 67.4 & 67.4 & 71.1 & 71.3 & 83.7 & \\
\hline \hline
\end{tabular}

${ }^{\mathrm{a} B a s e d}$ upon Ref. 12.
TABLE IX. Deviations from LSD of the curvature energy $\gamma$ of jellium in various density functional approaches and in DMC fit, for LSD densities. For an estimate of $\gamma^{\mathrm{LSD}}$, see Table VIII of Ref. 16.

\begin{tabular}{lcccc}
\hline \hline & \multicolumn{4}{c}{$\gamma_{\mathrm{xc}}-\gamma_{\mathrm{xc}}^{\mathrm{LSD}}$ (millihartree/bohr) } \\
$r_{s}$ & BLYP & PBE & PKZB & DMC fit \\
\hline 1.00 & -1.78 & -1.49 & -4.36 & -5.85 \\
2.00 & -0.76 & -0.33 & -1.04 & -1.15 \\
3.25 & -0.29 & -0.09 & -0.35 & -0.35 \\
4.00 & -0.17 & -0.05 & -0.21 & -0.26 \\
5.62 & -0.06 & -0.02 & -0.09 & -0.20 \\
\hline \hline
\end{tabular}

functionals should produce no relative error in $\sigma_{\mathrm{xc}}$, and we expect that the sophisticated RPA + would show the most correct approach to this limit. The $r_{s} \rightarrow \infty$ limit is stronglycorrelated, so the PKZB meta-GGA might be most trustworthy ${ }^{30}$ in that limit.

Surface energies $\sigma_{\mathrm{xc}}$ are typically calculated for a few values of $r_{s}$. It is thus useful to have a formula to interpolate and extrapolate to other $r_{s}$. We propose one which has four fit parameters;

$$
\sigma_{\mathrm{xc}}\left(r_{s}\right)=\frac{A}{r_{s}^{7 / 2}\left(1+B x+C x^{2}+D x^{3}\right)},
$$

where

$$
x=\left(1+r_{s}\right)^{1 / 2}-1 .
$$

The small- $r_{s}$ limit $\left[\sim r_{s}^{-7 / 2}+O\left(r_{s}^{-5 / 2}\right)\right]$ of Eq. (15) follows by applying the density functionals to the Thomas-Fermi density profile $\left(3 / 4 \pi r_{s}^{3}\right) f\left(x / r_{s}^{1 / 2}\right)$, treating $\ln r_{s}$ as a constant. The large- $r_{s}$ limit of Eq. (15) $\left[\sim r_{s}^{-5}+O\left(r_{s}^{-11 / 2}\right)\right]$ was chosen because it gave a good fit, and is consistent with a limiting density profile $\left(3 / 4 \pi r_{s}^{3}\right) g\left(r_{s} x\right)$; however, it may only reflect the disappearance (Table II of Ref. 31) of the density tail outside the positive-background edge in this limit. A formula like Eq. (15) for $\epsilon_{\mathrm{xc}}^{\text {unif }}\left(r_{s}\right)$ (but including $\ln r_{s}$ contributions) was presented in Ref. 32. Table X confirms that this simple formula works well. When fitted in the range 2.07 $<r_{s}<4.00$, it makes an interpolation error of $<0.1 \%$, while its extrapolation error to the wider range $0.8<r_{s}<6.00$ is only $1 \%$ or less. [Note that Eq. (15) can be rearranged so that $A, B, C, D$ can be found by solving four simultaneous linear equations.]

The parameters for Eq. (15) are shown in Table XI. Note that the parameter $A$ is nearly the same for LSD, PBE, $\mathrm{PKZB}$, and RPA+, as expected, since all these functionals have the same correct small- $r_{s}$ limit. On the other hand, the parameter $A$ for DMC or DMC fit is rather different, reflecting the greater imprecision of those surface energies.

\section{CONCLUSIONS}

The BLYP GGA, which gives accurate energies for atoms and molecules, is unsatisfactory for jellium clusters because it does not yield the correct correlation energy for a uniform 
TABLE X. Jellium surface exchange-correlation energies $\sigma_{\mathrm{xc}}$, for LSD densities. The symbol * denotes interpolated and extrapolated values calculated from Eq. (15). The parameters were found using the known surface energies at $r_{s}=2.07,2.66,3.28,4.00$.

\begin{tabular}{lcccccccc}
\hline \hline & & & \multicolumn{7}{c}{$\sigma_{\mathrm{xc}}\left(\mathrm{erg} / \mathrm{cm}^{2}\right)$} \\
$r_{s}$ & LSD & LSD* & PBE & PBE* & PKZB & PKZB* & ${\text { RPA }{ }^{\mathrm{a}}}^{*}$ & $\mathrm{RPA}^{*} *$ \\
\hline 0.80 & 91706 & 92444 & 90617 & 91555 & 92891 & 93917 & & 93398 \\
1.00 & 40928 & 41291 & 40276 & 40715 & 41463 & 41933 & & 41718 \\
2.00 & 3357 & 3357 & 3263 & 3267 & 3400 & 3404 & 3413 & 3414 \\
2.07 & 2962 & 2962 & 2881 & 2881 & 3004 & 3004 & 3015 & 3015 \\
2.30 & 2019 & 2019 & 1960 & 1961 & 2047 & 2047 & 2060 & 2059 \\
2.66 & 1188 & 1188 & 1151 & 1151 & 1204 & 1204 & 1214 & 1214 \\
3.00 & 763.9 & 763.9 & 739.1 & 739.0 & 774.4 & 774.4 & 781 & 782.1 \\
3.25 & 568.6 & 568.5 & 549.5 & 549.5 & 576.4 & 576.4 & & 582.4 \\
3.28 & 549.5 & 549.5 & 531.1 & 531.1 & 557.1 & 557.1 & 563 & 563.0 \\
4.00 & 261.7 & 261.7 & 252.5 & 252.5 & 265.6 & 265.6 & 268 & 268.0 \\
5.00 & 111.5 & 111.2 & 107.2 & 107.1 & 113.2 & 113.0 & 113 & 113.0 \\
5.62 & 70.0 & 70.0 & 67.4 & 67.4 & 71.3 & 71.3 & & 70.6 \\
6.00 & 53.6 & 53.8 & 51.6 & 51.8 & 54.6 & 54.8 & 54 & 53.9 \\
\hline \hline
\end{tabular}

${ }^{\mathrm{a}}$ Reference 24.

electron gas. Table II shows how the BLYP GGA improves as $r_{s} \rightarrow 0$, i.e., as the 20 -electron cluster becomes a Ca atom ${ }^{10}$ (with a self-interacting nucleus). But the LSD, PBE GGA, and PKZB meta-GGA are all rather accurate for jellium clusters. The most accurate density functional results overall are those of the meta-GGA, which satisfies the most exact constraints and works best for a wide range of systems including atoms, molecules, solids, and surfaces. ${ }^{2}$

We have presented and confirmed a method [Eq. (14)] for the extraction of accurate surface and curvature energies from the energies of finite clusters. The same method presumably would work even better for the extraction of surface energies from planar slabs of finite thickness, since for slabs the curvature energy term of Eq. (14) would be absent. For a closed-shell slab of background thickness $L$ and crosssectional area $A$, we could write

$$
\frac{E}{A}-\frac{E^{\mathrm{LSD}}}{A}=\left(\alpha-\alpha^{\mathrm{LSD}}\right) L+2\left(\sigma-\sigma^{\mathrm{LSD}}\right)
$$

TABLE XI. Parameters of Eq. (15) used to calculate interpolated and extrapolated values (*) of Table X and DMC fit of Table VIII. For the small- $r_{s}$ limit of $\sigma_{\mathrm{xc}}$, we independently estimate $A$ $=50000 \pm 500 \mathrm{erg} / \mathrm{cm}^{2}$ by linearly extrapolating $\left(\sigma-\sigma_{T F}\right) r_{s}^{7 / 2}$ as a function of $r_{s}$ to $r_{s}=0$, using $\sigma$ from Table I of Ref. 31 and the Thomas-Fermi $\sigma_{T F}$ from Eq. (8.15) of Ref. 33. Note that the DMC fit parametrization, fitted at $r_{s}=2.00,3.25,4.00$, and 5.62, shows a singularity for $r_{s}>12$.

\begin{tabular}{lccccc}
\hline \hline $\begin{array}{l}\text { param- } \\
\text { eter }\end{array}$ & LSD & PBE & PKZB & RPA+ & $\begin{array}{c}\text { DMC } \\
\text { fit }\end{array}$ \\
\hline$A$ & 50695 & 51936 & 51565 & 52227 & 47875 \\
$B$ & 0.74651 & 0.89526 & 0.74719 & 0.87924 & 0.35215 \\
$C$ & -0.57888 & -0.66994 & -0.56519 & -0.79810 & 0.09153 \\
$D$ & 0.25146 & 0.27742 & 0.24187 & 0.34685 & -0.10819 \\
\hline \hline
\end{tabular}

fitting both $\alpha-\alpha^{\mathrm{LSD}}$ and $\sigma-\sigma^{\mathrm{LSD}}$ to $\left(E-E^{\mathrm{LSD}}\right) / A$ as a function of $L$. Given $\alpha$, an alternative accurate way to extract $\sigma$ from slab calculations is also known. ${ }^{34}$

The correction to the LSD energy can be remarkably simple and insensitive to details of the electron density. That is the idea behind our Eq. (14), and also behind the way Mattsson and Kohn ${ }^{35}$ proposed to correct LSD or GGA surface energies. For metal surfaces, wave vector analysis ${ }^{13,36}$ provides a partial explanation for the success of this idea, but its applicability may be much wider, as found here; see also Fig. 3 of Ref. 17 for closed-shell fullerenes.

When we extract the jellium surface energy from the diffusion Monte Carlo energies of jellium spheres, ${ }^{10}$ we obtain in Table VIII values that are close to those of the PKZB meta-GGA and slightly higher than those of LSD. Unlike Refs. 12 and 21, but like Refs. 13, 24, and 10, we find no evidence that the density functionals are seriously in error for the surface energy. We have also proposed a formula [Eq. (15)] for the interpolation and extrapolation of the $r_{s}$-dependence of the surface energy. This formula should be used for extrapolation only when the input $\sigma_{\mathrm{xc}}\left(r_{s}\right)$ are highly precise, as they are in our LSD, PBE, PKZB, and RPA+ calculations.

Our analysis brings the jellium surface energies from the diffusion Monte Carlo method into much closer agreement with those from three sophisticated methods which produce values for $\sigma_{\mathrm{xc}}$ that agree within 1\%: (1) the PKZB metageneralized gradient approximation; ${ }^{2}$ (2) a GGA short-rangecorrelation correction to the random phase approximation $(\mathrm{RPA}+) ;^{24}$ and (3) a wave-vector-interpolation (WVI) longrange correction to the PBE GGA for exchange and correlation. $^{13}$

According to Table VIII, the PBE GGA surface energies are slightly lower and thus less accurate than the LSD surface energies. In fact the PBE gradient corrections to LSD 
improve the energies of small clusters while worsening those of large clusters, as can be seen in Fig. 5.

To better understand this situation, let us recall that the cohesive or atomization energy $\epsilon_{\mathrm{coh}}$ and the monovacancy formation energy $\epsilon_{\mathrm{vac}}$ of a simple monovalent metal are, respectively, ${ }^{14}$

$$
\begin{aligned}
& \epsilon_{\mathrm{coh}} \simeq 4 \pi r_{s}^{2} \sigma+2 \pi r_{s} \gamma, \\
& \epsilon_{\mathrm{vac}} \simeq 4 \pi r_{s}^{2} \sigma-2 \pi r_{s} \gamma,
\end{aligned}
$$

the energies to create the positively-curved surface of an atom or the negatively-curved surface of a monovacancy. Let us focus on Eq. (17). In LSD, $\sigma$ is nearly correct but $\gamma$ is too large, leading to bulk overbinding of atoms, i.e., to a cohesive energy that is too large. In PBE GGA, $\sigma$ is lower and less accurate than in LSD, while $\gamma$ is not so different from its LSD value, so these errors in Eq. (17) tend to cancel, producing an accurate cohesive energy for the wrong reason in PBE GGA. In PKZB meta-GGA, $\sigma$ is slightly higher and thus more accurate than in LSD, while $\gamma$ is significantly lower and more accurate than LSD, producing the right cohesive energy for the right reason. Because the bulk of a monovalent metal should be treated accurately by any of these approximations, the error of the cohesive energy is essentially the self-interaction error, i.e., a failure of the functional to describe properly a one-electron density. Thus the proper self-interaction correction to LSD for a one-electron atom is in the curvature energy, not in the surface energy. (These observations are also consistent with the performance of the density functionals for the atomization energies of real solids and of molecules. ${ }^{2}$ ) We would then also expect accurate vacancy formation energies ${ }^{14,37}$ from the PKZB metaGGA.

To some extent, we can understand why the functionals perform as they do. The negative second-order gradient coefficient for exchange is too large in PBE and other GGA's by almost a factor of two, making $\sigma_{x}$ and thus $\sigma_{\mathrm{xc}}$ somewhat too low. The PKZB meta-GGA, which has the correct firstprinciples gradient coefficient, improves the surface energy as a result. The PKZB meta-GGA also improves the curvature energy, probably because of its use of the extra ingredients $\tau_{\uparrow}(\mathbf{r})$ and $\tau_{\downarrow}(\mathbf{r})$. Since the second-order gradient expansion of $\tau$ includes $\nabla^{2} n$ as well as $n^{5 / 3}$ and $|\nabla n|^{2} / n$ terms, it is also fair to say that the prediction of the right curvature energy requires the use of the Laplacian of the density.

\section{ACKNOWLEDGMENTS}

We are grateful to F. Sottile and P. Ballone for providing their results prior to publication and useful discussions. This work was supported in part by the National Science Foundation under Grant Nos. DMR-9810620 and DMR-0135678.
*Electronic address: 1ma@ fis.ua.pt; Also at Center for Computational Physics, University of Coimbra, 3004-516 Coimbra, Portugal.

†Electronic address: perdew@tulane.edu

†Electronic address: tcarlos@teor.fis.uc.pt

${ }^{1}$ W. Kohn and L.J. Sham, Phys. Rev. 140, A1133 (1965).

${ }^{2}$ S. Kurth, J.P. Perdew, and P. Blaha, Int. J. Quantum Chem. 75, 889 (1999).

${ }^{3}$ M. Brack, Rev. Mod. Phys. 65, 677 (1993).

${ }^{4}$ J.P. Perdew and Y. Wang, Phys. Rev. B 45, 13244 (1992).

${ }^{5}$ D.M. Ceperley and B.J. Alder, Phys. Rev. Lett. 45, 566 (1980).

${ }^{6}$ A.D. Becke, Phys. Rev. A 38, 3098 (1988).

${ }^{7}$ C. Lee, W. Yang, and R.G. Parr, Phys. Rev. B 37, 785 (1988).

${ }^{8}$ J.P. Perdew, K. Burke, and M. Ernzerhof, Phys. Rev. Lett. 77, 3865 (1996).

${ }^{9}$ J.P. Perdew, S. Kurth, A. Zupan, and P. Blaha, Phys. Rev. Lett. 82, 2544 (1999).

${ }^{10}$ F. Sottile and P. Ballone, Phys. Rev. B 64, 045105 (2001).

${ }^{11}$ P. Ballone, C.J. Umrigar, and P. Delaly, Phys. Rev. B 45, 6293 (1992)

${ }^{12}$ P.H. Acioli and D.M. Ceperley, Phys. Rev. B 54, 17199 (1996).

${ }^{13}$ Z. Yan, J.P. Perdew, S. Kurth, C. Fiolhais, and L. Almeida, Phys. Rev. B 61, 2595 (2000); 64, 049904 (2001).

${ }^{14}$ J.P. Perdew, Y. Wang, and E. Engel, Phys. Rev. Lett. 66, 508 (1991); [The liquid drop model for nuclei was proposed by C.F. von Weizsaecker, Z. Phys. 96, 431 (1935)].

${ }^{15}$ N.D. Lang and W. Kohn, Phys. Rev. B 1, 4555 (1970).

${ }^{16}$ C. Fiolhais and J.P. Perdew, Phys. Rev. B 45, 6207 (1992).

${ }^{17}$ A.J. Williamson, R.Q. Hood, and J.C. Grossman, Phys. Rev. Lett. 87, 246406 (2001).
${ }^{18}$ L. Fan and T. Ziegler, J. Chem. Phys. 94, 6057 (1991).

${ }^{19}$ L. M. Almeida, C. Fiolhais, and M. Causà, Int. J. Quantum Chem. (to be published).

${ }^{20}$ M. Madjet, C. Guet, and W.R. Johnson, Phys. Rev. A 51, 1327 (1995).

${ }^{21}$ E. Krotscheck and W. Kohn, Phys. Rev. Lett. 57, 862 (1986).

${ }^{22}$ G. Ortiz and P. Ballone, Phys. Rev. B 50, 1391 (1994).

${ }^{23}$ P. Gori-Giorgi, F. Sacchetti, and G.B. Bachelet, Phys. Rev. B 61, 7353 (2000).

${ }^{24}$ Z. Yan, J.P. Perdew, and S. Kurth, Phys. Rev. B 61, 16430 (2000).

${ }^{25}$ J.C. Boettger, J.R. Smith, U. Birkenheuer, N. Roesch, S.B. Trickey, J.R. Sabin, and S.P. Apell, J. Phys.: Condens. Matter 10, 893 (1998).

${ }^{26}$ V. Fiorentini and M. Methfessel, J. Phys.: Condens. Matter 10, 895 (1998)

${ }^{27}$ F. Furche, Phys. Rev. B 64, 195120 (2001).

${ }^{28}$ M. Lein, E.K.U. Gross, and J.P. Perdew, Phys. Rev. B 61, 13431 (2000).

${ }^{29}$ J.M. Pitarke and J.P. Perdew (unpublished).

${ }^{30}$ M. Seidl, J.P. Perdew, and S. Kurth, Phys. Rev. A 62, 012502 (2000).

${ }^{31}$ J.P. Perdew and Y. Wang, Phys. Rev. B 38, 12228 (1988).

${ }^{32}$ M. Seidl and J.P. Perdew (unpublished).

${ }^{33}$ N.D. Lang, Solid State Phys. 28, 225 (1973).

${ }^{34}$ J.M. Pitarke and A.G. Eguiluz, Phys. Rev. B 57, 6329 (1998); 63, 45116 (2001).

${ }^{35}$ A.E. Mattsson and W. Kohn, J. Chem. Phys. 115, 3441 (2001).

${ }^{36}$ D.C. Langreth and J.P. Perdew, Phys. Rev. B 15, 2884 (1977).

${ }^{37}$ K. Carling, G. Wahnström, T.R. Mattsson, A.E. Mattsson, N. Sandberg, and G. Grimvall, Phys. Rev. Lett. 85, 3862 (2000). 